\title{
Cardiopulmonary Exercise Testing in Patients with Implantable Cardioverter- Defibrillator: A Retrospective Study
}

\author{
Carolina Mizzaci, ${ }^{\oplus}$ Thiago Fagundes, ${ }^{\circledR}$ Felipe Malafaia, ${ }^{\circledR}$ Sandro Felicioni, ${ }^{\circledR}$ Susimeire Buglia, ${ }^{\circledR}$ Carlos Alberto \\ Hossri, ${ }^{\circledR}$ Almir Ferraz, ${ }^{\circledR}$ Rica Buchler, ${ }^{\oplus}$ Romeu Meneghelo, ${ }^{\circledR}$ Luiz Mastrocolla ${ }^{\odot}$ \\ Instituto Dante Pazzanese de Cardiologia, São Paulo, SP - Brazil
}

\section{Abstract}

Background: Cardiopulmonary exercise testing is widely used in the evaluation of patients with left ventricular dysfunction, and some of these patients have an implantable cardioverter-defibrillator (ICD). However, this test presents specific challenges because of the susceptibility to ventricular arrhythmias during maximal levels of exercise.

Objective: To evaluate the safety of cardiopulmonary exercise testing in patients with ICD.

Methods: The study included patients with ICD who underwent cardiopulmonary exercise testing between 2007 and 2015. The tests were completed once the electronic devices were programmed. The maximum allowed heart rate reached during exercise was 10 beats below the first therapy zone programmed.

Results: The study included 69 patients with mean age $53.7 \pm 10.8$ years, including $68 \%$ men. Exercise time was $8.7 \pm 2.3$ minutes, with peak oxygen consumption of $13.3 \pm 4.3 \mathrm{ml} . \mathrm{kg}^{-1} \cdot \mathrm{min}^{-1}$. Peak heart rate was $62.9 \pm 13.4 \%$ of the maximum rate predicted, with all patients taking specific medication. Ventricular arrhythmia was observed in $29 \%$ of the patients, and paired ventricular extrasystoles, ventricular bigeminism or non-sustained ventricular tachycardia were observed in only $14.5 \%$ of the patients. There was no sustained ventricular arrhythmia resulting in ICD therapy or other complications, such as inappropriate therapies. The frequency of severe events was $0 \%$, $95 \%$ CI $(0-5.2 \%)$.

Conclusion: In the sample of patients evaluated, the cardiopulmonary exercise testing was shown to be safe during its performance in a hospital setting, following the safety standards. (Int J Cardiovasc Sci. 2019;32(4):368-373)

Keywords: Cardiovascular Diseases; Sudden Cardiac Death; Left Ventricular Dysfunction; Breathing Exercise; Defibrillators, Implantable; Arrhythmias, Cardiac.

\section{Introduction}

Sudden cardiac death (SCD) is a major problem in patients with cardiovascular disease and it is mostly caused by ventricular arrhythmia. ${ }^{1}$ The pathophysiology of SCD involves an electrical instability event with induction of ventricular tachycardia (VT), which leads to ventricular fibrillation (VF) in 80 to $85 \%$ of the cases. Since the first report of implantable cardioverter- defibrillator (ICD) implantation in 1980, treatment of ventricular tachyarrhythmia underwent major changes. Initially used in patients surviving SCD, ICD was also recommended as a preventive therapy in patients at high risk for arrhythmic events. ${ }^{1-5}$

Cardiopulmonary exercise testing (CPET) adds information on ventilatory dynamics and gas exchange during exercise, allowing greater precision in the evaluation of the aerobic functional capacity of individuals 
with ICD. It is equally important in patients with left ventricular dysfunction, for prognostic evaluation, therapeutic control, prescription of physical activity and indication of cardiac transplantation. ${ }^{6}$

In patients with ICD, CPET should be preferably performed in a hospital setting. It is essential to be aware of basic parameters and implantable device programming. The physician should be aware of the heart rate zones where ICD therapies are programmed, in order to avoid inappropriate therapies. This type of therapy is harmful to the patient, due to its psychological and clinical implications. ${ }^{7,8}$

Literature data demonstrate that symptom-limited exercise testing causes ventricular extrasystole in more than half of patients with coronary artery disease. ${ }^{9-11}$ However, 20\% of exercise-induced arrhythmias are manifested as paired ventricular extrasystoles or nonsustained VT. ${ }^{9}$ Young et. al., ${ }^{12}$ reported complications, such as VT, VF and bradycardia, requiring immediate medical treatment in 24 out of 263 patients $(9.1 \%)$ at high risk for ventricular arrhythmias during maximal exercise testing. In another study in which 107 exercise tests were evaluated in individuals with ICD, ventricular arrhythmias requiring therapy or death ${ }^{13}$ were not observed.

Few data are available on the responses and complications associated with CPET in patients with ICD. This study aimed at evaluating the incidence of complications during this test in this population. To do this, the occurrence of ventricular arrhythmias (isolated extrasystoles, paired extrasystoles, ventricular bigeminism and ventricular tachycardia), appropriate therapies, inappropriate therapies, cardiorespiratory arrest during or immediately after exercise, hemodynamic instability, need for hospital admission due to cardiovascular complications and death were observed. Besides, other variables such as metabolic, ventilatory and cardiovascular response, symptoms, duration of exercise achieved and maximal oxygen consumption $\left(\mathrm{VO}_{2}\right)$ were also reported.

\section{Methodology}

This is a retrospective unicentric observational study conducted between 2007 and 2015, including which male and female patients with ICD for more than three months, for primary or secondary prevention of SCD, who underwent cardiopulmonary exercise testing as recommended by the attending physician.
CPET was performed using a calibrated breath by breath gas analyzer, Ultima, Medical Graphic Corporation (MGC), performed according to traditional guidelines. Continuous 12-lead electrocardiographic monitoring and recording system modified by Mason and Likar, Tecnologia Eletrônica Brasileira (TEB), model APEX 2000, was used throughout the test. Measurement of arterial saturation was performed by pulse oximetry (CMS50D) and blood pressure measurement by aneroid sphygmomanometer (Welch Allyn). In addition, latex insulating gloves were used by the medical and technical team throughout the test in order to avoid potential electrical therapies. A magnet was available in the test room in case of inappropriate ICD therapies.

The patients were submitted to ICD programming analysis by means of telemetry prior to the CPET. Telemetry was performed using specific equipment related to the manufacturer of the device, in order to obtain detailed programming information, such as VT and VF zones, and the relevant therapies. No therapy was changed or disabled before the test.

For each patient, a heart rate cutoff value was determined, based on individual device programming. The maximum heart rate established for the protocols applied during exercise was 10 beats below the first VT zone (VT1) programmed in the ICD. In the VT1 zone, established during ICD programming, usually only the monitoring of electrocardiographic events occurs. In the second VT or VT2 zone, there are three usual extra-stimulus series and in case of non-reversal, analyzed by the device algorithm, sequential joule discharges are automatically applied. In the VF zone, series of joule discharges are triggered, aiming at arrhythmia interruption.

The tests were performed with ramp protocols individually applied according to the estimated functional capacity of each patient, physician's experience and predicted $\mathrm{VO}_{2}$ calculated using the Wasserman algorithm. ${ }^{14}$

Traditionally, the criteria used are exhaustion, dyspnea, angina or dizziness, pathological abnormalities in blood pressure, sustained ventricular arrhythmia, orthopedic complaints and, in patients with interpretable rest electrocardiogram, ST segment depression greater than $3.0 \mathrm{~mm}$ or ST segment elevation greater than $1.0 \mathrm{~mm}$. All tests would be discontinued if the heart rate reached 10 beats below the programmed VT1 zone as a safety measure intended to avoid inappropriate therapies. 
Data analysis was performed by IBM SPSS software version 19 and Microsoft Office Excel 365 was used for data tabulation. The quantitative variables were presented by mean and standard deviation and qualitative variables by absolute frequency and percentage, then descriptive analysis of the data was performed. A 95\% confidence interval $(95 \% \mathrm{CI})$ was calculated using the binomial test. To test whether the frequency of serious events was smaller than $10 \%$, exact binomial test was used.

\section{Results}

In this study, 69 patients with ICD were included. Table 1 shows the characteristics of the study population.

Exercise time was $8.7 \pm 2.3$ minutes, with peak $\mathrm{VO}_{2}$ of $13.3 \pm 4.3 \mathrm{ml} \cdot \mathrm{kg}^{-1} \cdot \mathrm{min}^{-1}$. The heart rate reached at the peak of exercise was $105.9 \pm 22.9$ beats per minute (bpm), corresponding to $62.9 \pm 13.4 \%$ of the upper limit for the predicted age. All tests were discontinued due to patient exhaustion. Mean final speed was $3.9 \pm 0.9 \mathrm{~km} / \mathrm{h}$, while mean final inclination was $11.2 \pm 3.1 \%$ in this protocol. The other CPET variables are presented in Table 2.

The heart rate programmed for the VT1 zone in the ICD was $150 \pm 9.1 \mathrm{bpm}$ on average. During exercise, the patients achieved $71.8 \%$ of the heart rate programmed in the VT1 zone, $61.7 \%$ in the VT2 zone and $53.4 \%$ in the VF zone.

Chart 1 shows the arrhythmias found in the study. The following were considered as complex arrhythmias: paired ventricular extrasystoles, ventricular bigeminism, ventricular tachycardia. There were no sustained ventricular arrhythmia resulting in ICD therapy or inappropriate therapies. Also, there was no cardiorespiratory arrest, hemodynamic instability during or immediately after exercise or hospital admissions due to cardiovascular complications or death. The frequency of severe events was $0 \%, 95 \% \mathrm{CI}$ $(0-5.2 \%)$. Thus, we have that the frequency of serious events will be less than $10 \%$.

\section{Discussion}

CPET is a fundamental test recommended by consensus for the evaluation of patients with left ventricular dysfunction, both for prognostic characterization and for therapeutic control and assistance in the indication for cardiac transplantation. ${ }^{15,16}$ However, there is little information regarding the safety of this test in individuals with ICD. As we know, CDI therapy has become a

\begin{tabular}{lc}
\hline $\begin{array}{l}\text { Table 1 - Baseline characteristics of the study } \\
\text { population }\end{array}$ & Value \\
\hline Variable & $55 \pm 10$ \\
\hline Age (years) & \\
Gender (\%) & 68.1 \\
Male & 31.9 \\
Female & $25.5 \pm 4.8$ \\
BMI (kg/m²) & \\
Diagnosis (\%) & 40.6 \\
Ischemic cardiomyopathy & 56.5 \\
Dilated cardiomyopathy & 2.9 \\
Hypertrophic cardiomyopathy & \\
Resting ECG (\%) & \\
PMI: body mass index; ECG: electrocardiogram; ACEI: angiotensin- \\
Pacemaker rhythm
\end{tabular}

standard indication in this population, both for primary and secondary prevention. ${ }^{1,3,4}$ Therefore, the importance of this study was to check the safety of CPET in this type of population.

The major concern in performing CPET in patients with ICD is the risk of complications such as ventricular arrhythmias during exercise, resulting in therapies by the device. ${ }^{7,17}$ Another risk inherent in the procedure is that it triggers inappropriate therapies by the implantable electronic device. It is known that unnecessary shocks should be avoided, while rapid appropriate therapy for ventricular tachyarrhythmia should be affordable. Inappropriate ICD therapy is not only traumatic for 


\section{Table 2 - Variables analyzed during the CPET}

\begin{tabular}{|c|c|}
\hline Variable & Value \\
\hline $\mathrm{SBP}$ at rest $(\mathrm{mmHg})$ & $117.3 \pm 22.7$ \\
\hline $\mathrm{SBP}(\mathrm{mmHg})$ & $134.1 \pm 31.0$ \\
\hline HR (bpm) & $68.3 \pm 10.8$ \\
\hline Peak HR (bpm) & $105.9 \pm 23.0$ \\
\hline Peak $\mathrm{VO}_{2}\left(\mathrm{ml} \cdot \mathrm{min}^{-1}\right)$ & $973 \pm 361$ \\
\hline Peak $\mathrm{VO}_{2}\left(\mathrm{~mL} \cdot \mathrm{kg}^{-1} \cdot \mathrm{min}^{-1}\right)$ & $13.3 \pm 4.3$ \\
\hline Predicted $\mathrm{VO}_{2}(\%)$ & $49 \pm 16.7$ \\
\hline Peak LV (L.min $\left.{ }^{-1}\right)$ & $53.8 \pm 50$ \\
\hline Peak VE $/ \mathrm{VO}_{2}$ & $51 \pm 17.5$ \\
\hline Peak VE/ $\mathrm{VCO}_{2}$ & $45 \pm 14.4$ \\
\hline Slope VE/ $\mathrm{VCO}_{2}$ & $42.4 \pm 18.1$ \\
\hline Peak RER & $1.12 \pm 0.14$ \\
\hline Peak pulse $\mathrm{O}_{2}\left(\right.$ ml.beat $\left.^{-1}\right)$ & $10.3 \pm 3.5$ \\
\hline $\mathrm{T} 1 / 2$ (seconds) & $150 \pm 46.9$ \\
\hline OUES & $800 \pm 479$ \\
\hline
\end{tabular}

SBP: systolic blood pressure; HR: heart rate; $\mathrm{VO}_{2}$ : oxygen consumption; RER: respiratory exchange ratio; OUES: oxygen uptake efficiency slope; $\mathrm{VE} / \mathrm{VCO} \mathrm{O}_{2}$ : ventilatory equivalent for $\mathrm{CO}_{2} ; \mathrm{VE} / \mathrm{VO}_{2}$ : ventilatory equivalent for oxygen. patients but it is also associated with an increased risk of death. ${ }^{18}$

The ICDs present algorithms for the detection of ventricular tachyarrhythmia and discriminatory algorithms for supraventricular tachycardia. However, these may fail and result in inappropriate therapies, such as supraventricular tachycardia therapy. To avoid this risk, during CPET, it was established that the heart rate during exercise should have the zone of the first programmed therapy as a limit of attention. Hence the importance of prior knowledge of device programming by telemetry.

Although exercise may predispose to ventricular arrhythmia, which is common in heart failure, the overall frequency of arrhythmia during exercise testing is low. In this study, we demonstrated that CPET is safe because no severe arrhythmia has been identified. This is consistent with an earlier study by Chinnaiyan et al., ${ }^{13}$ who evaluated 84 patients (mean age $67 \pm 12$ years; $76 \%$ men). Participants underwent 107 stress tests, including 44 exercises and 63 pharmacological evaluations (22 dobutamine, 41 dipyridamole). No ICDs were inactivated before the test. Four patients presented nonsustained self-limited VT at the peak of stress. None of them had sustained VT requiring therapy. There were no deaths or hospital readmissions due to ventricular arrhythmias.

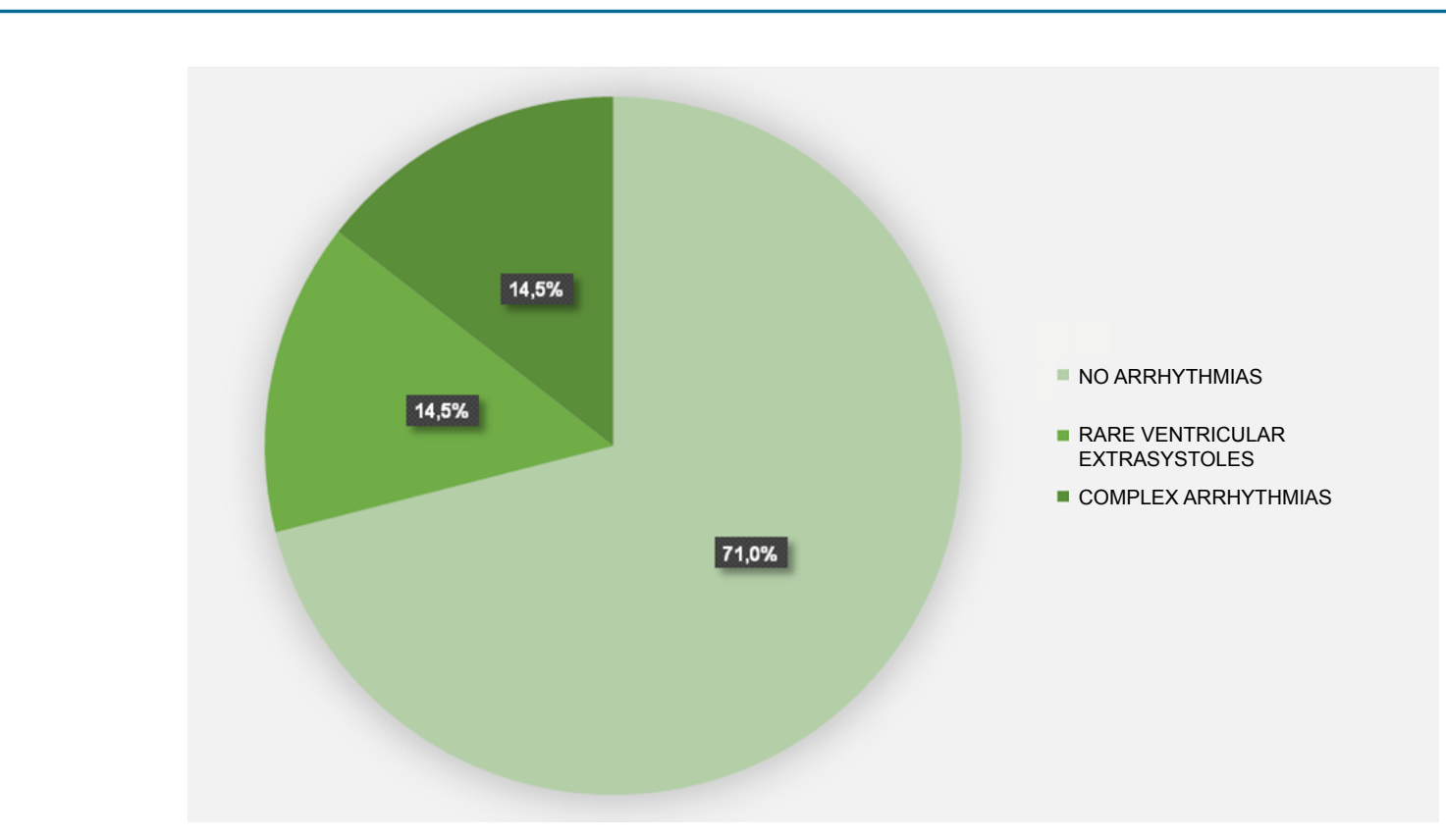

Chart 1 - Incidence of ventricular arrhythmias in the study population. 
Voss et al., ${ }^{19}$ also demonstrated the safety of exercise testing in 400 patients with ICD. Of these, 200 patients performed a ramp protocol with an initial load of $0 \mathrm{~W}$ increased by $15 \mathrm{~W}$ every minute. Another 200 patients with ICD had a slightly modified ramp protocol with an initial workload of $0 \mathrm{~W}$, but with capacity increased by $15 \mathrm{~W}$ every 2 minutes. The study population consisted mainly of patients with ischemic heart disease $(63 \%)$. Atrial fibrillation was present in $16 \%$ of the individuals. Left ventricular ejection fraction was $28 \% \pm 8$. In this cohort of patients, no sustained ventricular arrhythmia and no death occurred during or after the exercise testing. No inappropriate shock was observed.

In this study, during CPET, the heart rate of patients did not reach the device therapy zones, most likely because they were on negative chronotropic medications. Although safe heart rate limits were set for each patient, most tests were discontinued due to exhaustion before the heart rate limit was reached. Although the tests were discontinued at a heart rate below the ICD programming, it is observed that the mean RER was greater than 1.1, demonstrating maximum exercise. ${ }^{6}$

In the current study, ventricular arrhythmias were observed in $29 \%$ of the individuals, including paired ventricular extrasystoles, ventricular bigeminism or nonsustained VT in $14.5 \%$. However, there were no sustained ventricular arrhythmias that resulted in ICD therapy or inappropriate therapies. There was no cardiorespiratory arrest, hemodynamic instability during or immediately after exercise, or hospital admission due to cardiovascular complications. As an implication for clinical practice, CPET performed in patients with ICD was safe when performed in a hospital setting, with prior knowledge of device programming and respecting the heart rate of the therapy zones.

The limitations of this study refer to the small number of patients included which, to some extent, is justified by the fear of referring patients with implantable devices to perform CPET, and because it is a retrospective study. As implications for future research, further prospective studies with larger samples should be conducted in order to obtain more robust results.

\section{Conclusion}

CPET in patients with ICD is a safe procedure in terms of severe acute complications, with low incidence of ventricular arrhythmia. Physicians performing CPET in these specific patients must be aware of ICD settings to limit heart rate below the first programmed therapy zone. There is no need for changes in device programming, such as deactivation of therapies, prior to this test.

\section{Author contributions}

Conception and design of the research: Mizzaci CC, Meneghelo RS, Mastrocola LE. Acquisition of data: Mizzaci CC, Fagundes TTS, Malafaia FL, Felicioni SP, Buglia S, Hossri CAC, Ferraz AS, Buchler RDD. Analysis and interpretation of the data: Mizzaci CC, Fagundes TTS, Malafaia FL, Felicioni SP, Buglia S, Hossri CAC, Ferraz AS, Buchler RDD, Meneghelo RS, Mastrocola LE. Statistical analysis: Mizzaci CC, Malafaia FL, Felicioni SP, Buglia S, Hossri CAC, Ferraz AS, Buchler RDD. Writing of the manuscript: Mizzaci CC, Fagundes TTS, Malafaia FL, Felicioni SP, Buglia S, Hossri CAC, Ferraz AS, Buchler RDD, Meneghelo RS, Mastrocola LE. Critical revision of the manuscript for intellectual content: Mizzaci CC, Fagundes TTS, Meneghelo RS, Mastrocola LE.

\section{Potential Conflict of Interest}

No potential conflict of interest relevant to this article was reported.

\section{Sources of Funding}

There were no external funding sources for this study.

\section{Study Association}

This study is not associated with any thesis or dissertation work.

\section{Ethics approval and consent to participate}

This article does not contain any studies with human participants or animals performed by any of the authors. 


\section{References}

1. Moss AJ, Hall WJ, Cannom DS, Daubert JP, Higgins SL, Klein H, et al. Improved survival with an implanted defibrillator in patients with coronary disease at high risk for ventricular arrhythmia. Multicenter Automatic Defibrillator Implantation Trial Investigators. N Engl J Med. 1996;335(26):1933-40.

2. Moss AJ, Zareba W, Hall WJ, Klein H, Wilber DJ, Cannom DS, et al. Prophylactic implantation of a defibrillator in patients with myocardial infarction and reduced ejection fraction. N Engl J Med. 2002;346(12):877-83.

3. Connolly SJ, Gent M, Roberts RS, Dorian P, Roy D, Sheldon RS, et al. Canadian implantable defibrillator study (CIDS) : a randomized trial of the implantable cardioverter defibrillator against amiodarone. Circulation. 2000;101(11):1297-302.

4. Connolly SJ, Hallstrom AP, Cappato R, Schron EB, Kuck KH, Zipes DP, et al. Meta-analysis of the implantable cardioverter defibrillator secondary prevention trials. AVID, CASH and CIDS studies. Antiarrhythmics vs Implantable Defibrillator study. Cardiac Arrest Study Hamburg. Canadian Implantable Defibrillator Study. Eur Heart J. 2000;21(24):2071-8.

5. Mann DL,Zipes DP, Libby P,Bonow R, Braunwald E. Braunwald `s heart disease: a textbook of cardiovascular medicine .10th ed. Philadelphia:Elsevier;2015

6. Guazzi M, Arena R, Halle M, Piepoli MF, Myers J, Lavie CJ. 2016 Focused Update: Clinical Recommendations for Cardiopulmonary Exercise Testing Data Assessment in Specific Patient Populations. Circulation. 2016;133(24):e694-711.

7. Kelly TM. Exercise testing and training of patients with malignant ventricular arrhythmias. Med Sci Sports Exerc. 1996;28(1):53-61.

8. Pinski SL, Fahy GJ. The proarrhythmic potential of implantable cardioverter-defibrillators. Circulation. 1995;92(6):1651-64.

9. Ryan M, Lown B, Horn H. Comparison of ventricular ectopic activity during 24-hour monitoring and exercise testing in patients with coronary heart disease. N Engl J Med. 1975;292(5):224-9.
10. Hermes IL, Marianna GS, Jessica RC, Carlos BR, Rafael CD, María Dolores RS, et al. Development and validation of a risk calculator predicting exercise-induced ventricular arrhythmia in patients with cardiovascular disease. Int J Cardiol. 2016;220:625-8.

11. Irace L, Agretto A, Vitale N, De Cicco D, Nave C, Vitale P. [Ergometric test in the diagnostic and prognostic evaluation of arrhythmia]. Minerva Cardioangiol. 1991;39(7-8):261-6.

12. Young DZ, Lampert S, Graboys TB, Lown B. Safety of maximal exercise testing in patients at high risk for ventricular arrhythmia. Circulation. 1984;70(2):184-91.

13. Chinnaiyan KM, Trivax J, Franklin BA, Williamson B, Kahn JK. Stress testing in patients with implantable cardioverter-defibrillators: a preliminary report. Prev Cardiol. 2007;10(2):92-5.

14. Wasserman K, Hansen JE, Sue DY, Stringer WW, Whipp BJ. Principles exercise testing and interpretation. 4th ed. Philadelphia: Lippincott Williams \& Wilkins; 2005. p. 80-1, 160-7.

15. Metra M, Dei Cas L, Panina G, Visioli O. Exercise hyperventilation chronic congestive heart failure, and its relation to functional capacity and hemodynamics. Am J Cardiol. 1992;70(6):622-8.

16. Bard RL, Gillespie BW, Clarke NS, Egan TG, Nicklas JM. Determining the best ventilatory efficiency measure to predict mortality in patients with heart failure. J Heart Lung Transplant. 2006;25(5):589-95.

17. Pashkow FJ, Schweikert RA, Wilkoff BL. Exercise testing and training in patients with malignant arrhythmias. Exerc Sport Sci Rev. 1997;25:235-69.

18. Satoh A, Niwano S, Niwano H, Kamiya K, Kishihara J, Aoyama Y, et al. Prediction of inappropriate implantable cardioverter-defibrillator therapies through parameters obtained in a simple exercise stress test. Int Heart J. 2012;53(5):276-81.

19. Voss F, Schueler M, Lauterbach M, Bauer A, Katus HA, Becker R. Safety of symptom-limited exercise testing in a big cohort of a modern ICD population. Clin Res Cardiol. 2016;105(1):53-8. 